\title{
Short versus long course antibiotic therapy for acute pyelonephritis in adults: a systematic review and meta-analysis
}

\author{
Franco Berti, ${ }^{1}$ Tiziana Marcella Attardo, ${ }^{2}$ Salva Piras, ${ }^{3}$ Letizia Tesei, ${ }^{4}$ Daniela Tirotta, ${ }^{5}$ Michela Tonani, ${ }^{6}$ \\ Greta Castellini, ${ }^{7,8}$ Silvia Gianola, ${ }^{8,9}$ Silvia Minozzi ${ }^{10}$
}

${ }^{1}$ Internal Medicine 2 Department, S. Camillo Forlanini Hospital, Roma; ${ }^{2}$ Internal Medicine Department, Hospital of Canicattì (AG); ${ }^{3}$ Internal Medicine Department, Civile Hospital, Alghero (SS); ${ }^{4}$ Internal Medicine Department, A.V. 2 Ancona, ASUR Marche; ${ }^{5}$ Internal Medicine Department, Cervesi Hospital, AUSL Romagna; ${ }^{6}$ Internal Medicine 2 Department, IRCCS Policlinico San Matteo, Pavia; ${ }^{7}$ Unit of Clinical Epidemiology, IRCCS Galeazzi Orthopedic Institute, Milano; ${ }^{8}$ Department of Biomedical Sciences for Health, University of Milano; ${ }^{9}$ Center of Biostatistics for Clinical Epidemiology, School of Medicine and Surgery, University of Milano-Bicocca, Monza (MB); ${ }^{10}$ Department of Epidemiology, Lazio Regional Health Service, Roma, Italy

\begin{abstract}
Acute pyelonephritis $(\mathrm{aPN})$ is defined as a severe form of urinary tract infection. Despite its severity and the high incidence in the community setting, there is no consensus on the optimal duration of treatment. The aim was to compare effectiveness and tolerability of short- versus long-course treatment with the same antibiotic agent in patients with aPN. We searched MEDLINE (PubMed), EMBASE and CENTRAL up to June 2016 for randomized controlled trials (RCTs). Three pairs of authors independently extracted data and appraised risk of bias. We included 4 RCTs (439 participants). Short antibiotic treatment lasted from 4 to 14 days and long treatment from 7 to 42 days but was at least 2 days longer than the corresponding short-course. At the end of treatment, we did not find any significant differences in clinical success [risk ratio (RR) 1.01; 95\% confidence interval (CI), 0.96-1.07, moderate quality evidence] as well as in microbiological success (RR 0.99; 95\% CI, 0.92-1.07, very low-quality evidence). At 4-6 weeks after the end of treatment there were no significant differences in clinical relapses (RR $1.20,95 \%$ CI 0.43-3.30, very low-quality evidence) and re-infection of other germs (RR 2.40; 95\% CI, 0.68-8.49, very low-quality evidence), even if short-term therapy seemed to have more risk of recurrences (RR 2.39, 95\% CI 1.19-4.83, very low quality of evidence). The incidence of any adverse effect seemed to be lower with the short-term therapy, though the results are not statistically significant (RR 0.63, 95\% CI 0.39-1.02, low quality evidence). Short-term treatment for aPN seems to be equivalent to long-term treatment in terms of clinical and microbiological success at the end of treatment or tolerability. The only relevant difference is the frequency of recurrence of the same biological germ up to 4-6 weeks after the end of treatment, which is significantly higher with the short-term therapy.
\end{abstract}

Correspondence: Franco Berti, Internal Medicine 2 Department, S. Camillo Forlanini Hospital, Circonvallazione Gianicolense 87, 00152 Roma, Italy.

Tel.: +39.06.37352792.

E-mail: fberti9@gmail.com

Key words: Urinary tract infection; drug resistance; treatment outcome; safety; pyelonephritis.

Conflict of interest: the authors declare no conflict of interest.

See online Appendix for additional material.

Received for publication: 14 January 2017.

Revision received: 25 October 2017.

Accepted for publication: 27 October 2017.

This work is licensed under a Creative Commons Attribution NonCommercial 4.0 License (CC BY-NC 4.0).

(C) Copyright F. Berti et al., 2018

Licensee PAGEPress, Italy

Italian Journal of Medicine 2018; 12:39-50

doi:10.4081/itjm.2018.840

\section{Introduction}

Acute pyelonephritis (aPN) is a severe form of urinary tract infection (UTI), which results from bacterial invasion of the renal parenchyma. The incidence of aPN is estimated at around 9-11 cases per 10,000 inhabitants and is four times more frequent in women than in men. ${ }^{1}$

Diagnosis is based on clinical criteria (fever, costovertebral pain, and dysuria symptoms) and microbiological criteria [pyuria and a positive urine culture with $\geq 10^{4}$ colony-forming units (cfu) per milliliter of urine]. ${ }^{2}$

The most common causative pathogen is Escherichia coli (70-90\% of uncomplicated UTIs and 21$54 \%$ of complicated UTIs). ${ }^{3}$ Other pathogens include Proteus species, Klebsiella species and Enterococci. ${ }^{4}$

Bacteria usually reach the kidneys by ascending from the lower urinary tract or, more rarely, through the bloodstream. ${ }^{5}$

Several factors may be involved in the etiology of aPN: anatomic or functional abnormalities of uri- 
nary tract drainage ${ }^{6}$ or metabolic disorders ${ }^{7,8}$ (intravenous drug abuse and endocarditis are implicated in gram-positive hematogenous infections).

Complicated aPN was defined as aPN occurring in any male patient, in patients with functional or anatomical abnormalities of the urinary tract, immunosuppressed persons, patients with a single kidney, permanent bladder catheter, nephrostomy or double-J catheter, or those patients who had experienced urinary tract manipulation in the previous two weeks. ${ }^{9}$

Pyelonephritis becomes a potentially fatal disease when secondary conditions develop, such as emphysematous pyelonephritis (20-80\% mortality rate), perinephric abscess (20-50\% mortality rate), or one of the sepsis syndromes $(>25 \%$ overall mortality rate). ${ }^{4}$ Pregnant women are more likely to develop pyelonephritis and pregnancy is associated with major risk of complications. ${ }^{10}$

The severity of aPN ranges from mild discomfort to life-threatening illness or death. ${ }^{11}$ Hospitalization may be required for up to $10-30 \%$ of cases. ${ }^{12}$

The extensive use of antibiotics has increased antibiotic resistance during recent years. ${ }^{13,14}$ For example Escherichia coli rates of resistance have reached $50 \%, 20 \%$, and $10 \%-20 \%$, respectively for ampicillin, fluoroquinolones, and trimethoprim/sulfamethoxazole (TMP-SMX). ${ }^{15}$ Given the high incidence of acute pyelonephritis in the community setting, measures should be taken to avoid further development of antimicrobial resistance. ${ }^{16}$

In the current International Clinical Practice Guidelines for the treatment of acute pyelonephritis, the recommended duration of treatment for pyelonephritis is 7 days for fluoroquinolones, 10-14 days for b-lactams and 14 days for TMP-SMX. ${ }^{17-19}$

No recommendation is provided for women or men hospitalized with acute pyelonephritis. ${ }^{20}$

The guidelines address only young, otherwise healthy women who are not pregnant. Best management for elderly individuals, men and patients with comorbidities remains unclear. Despite publication of the guidelines, studies demonstrate a wide variation in prescribing practices regarding the selection of antimicrobial agents and duration of therapy. ${ }^{21-23}$

A reduction in the duration of the administered regimes could minimize the selection pressure on potential pathogens, thereby reducing the risk for the emergence of new resistant strains. ${ }^{24}$

Under this perspective, we sought to compare short-course with long-course treatment with the same antibiotic regimes, administered by the same route and in the same total daily dosage for acute pyelonephritis, in terms of effectiveness and tolerability, by performing a systematic review and metaanalysis of randomized controlled trials (RCTs).

\section{Materials and Methods}

\section{Registered protocol}

We registered the present study in PROSPERO database (CRD42016051105).

\section{Inclusion criteria}

We included RCTs that compared a long-course versus a short-course antibiotic therapy of the same antibiotic agents administered by the same route and in the same total daily dosage. Long course was defined as a therapy, which lasts at least 2 days longer than the corresponding short-course treatment. The participants were eligible for inclusion if they were 18 years and older with acute PN, diagnosed based on clinical criteria (fever, costovertebral pain, and dysuria symptoms) and microbiologic criteria (pyuria and a positive culture with $>=104$ cfu per milliliter of urine. Pregnant women and patients, both hospitalized and outpatients, with anatomical or functional abnormalities of the urinary tract, permanent bladder catheter, immunosuppressed, oncological and diabetic were also considered. Trials with a mixed population were included in the systematic review if they provided separate data for aPN population.

\section{Types of outcome measures and follow-up assessment}

Our primary outcome was Clinical success, defined as a number of subjects with resolution of symptoms (fever, costovertebral pain and dysuria), signs (biohumoral tests as leukocyte count, inflammatory markers and renal function) and microbiologic criteria (pyuria and a positive culture with $\geq 10^{4}$ cfu per milliliter of urine) at the end of treatment.

Secondary outcomes were: Microbiological success, at the end of treatment defined as yielding sterile urine cultures or positive cultures with $<10^{3} \mathrm{cfu} / \mathrm{mL}$ of urine at the end of therapy; ${ }^{24}$ Clinical relapse at 4-6 weeks after the end of treatment, defined as the reappearance of signs and symptoms.

Microbiological relapse, defined as the reappearance of the original strain in a urine culture at 4-6 weeks after the end of treatment, based on species identification and serotyping results; Microbiological recurrence or reinfection, defined as the appearance of another bacteriologic strain in a urine culture at 46 weeks after the end of treatment, based on species identification and serotyping results; ${ }^{24}$ Renal impairment, defined as a glomerular fraction rate $<30$ $\mathrm{mL} / \mathrm{min}$ or creatinine increase $>50 \%$ from baseline level; Intensive Care Unit requirement; Mortality for any cause occurring during the study; Readmission for the same cause until the end of the follow-up period; Patients with any Adverse event defined as any unto- 
ward occurrence of any unfavorable and unintended clinically relevant medical sign, symptom or any disease temporally associated with the study, which did not necessarily have a causal relationship with the study procedure and patient withdrawals due to serious adverse events during the study.

\section{Search strategy}

We searched the following electronic databases: MEDLINE (PubMed) (January 1966 to June 2016), EMBASE (Elsevier, EMBASE.com) (January 1974 to June 2016) and CENTRAL (up June 2016) using the search strategy outlined in the Appendix.

We checked the reference lists of all studies included and of any systematic reviews we have identified during the search process.

We also searched the following clinical trial registries to identify ongoing trials: ClinicalTrials.gov (http://clinicaltrials.gov/) and Current Controlled Trials (http://www.controlledtrials.com/); we included studies written in English, French, Spanish and Italian.

\section{Selection of studies and data extraction}

Three pairs of authors (BF, ATM, PS, TL, TD, TM) screened titles and abstracts obtained by the search strategy. Then they independently assessed the full text of potentially relevant studies for inclusion. Any disagreement was solved by discussion with a further author (SM).

We adopted a standardized data collection form to extract the following information: number and characteristics of participants, setting, type of experimental and control intervention (antibiotic agent, length of treatment, any further treatment), length of followup, types of outcomes, country of origin, funding and conflict of interest, clinical success, bacteriologic efficacy, relapses, recurrences, any adverse events and/or patient withdrawals due to adverse events. We contacted authors if the reported data were insufficient or unclear.

\section{Risk of bias (quality) assessment}

Two authors (TL, TD) independently assessed the risk of bias of the included studies. Any disagreement was resolved by discussion with a further author (SM). We assessed the risk bias using the Cochrane criteria. ${ }^{25}$ We considered the following specific domains: sequence generation and allocation concealment (selection bias), blinding of participants and providers (performance bias), blinding of outcome assessors (detection bias), incomplete outcome data (attrition bias) and selective outcome reporting (reporting bias). Each domain was judged as high, low or unclear risk of bias. To incorporate our assessment of risk of bias in the review process, we first plotted the intervention effects estimates, stratified by risk of bias for allocation con- cealment (selection bias), blinding of outcome assessors (detection bias) and attrition bias. If differences in the results were present among studies at different risks of bias, we performed sensitivity analysis by excluding studies with high risk of bias from the analysis.

\section{Data synthesis}

We analyzed dichotomous outcomes by calculating the risk ratio (RR) for each trial with the uncertainty in each result being expressed with a $95 \%$ confidence interval (CI).

We combined the outcomes from the individual trials through meta-analysis where possible using a random-effects model $^{26}$ because a certain degree of heterogeneity was expected among trials. We analyzed heterogeneity by means of the $\mathrm{I}^{2}$ statistic and the $\mathrm{Chi}^{2}$ test. The cut-off points were $\mathrm{I}^{2}$ value of more than $50 \%$ and a $\mathrm{P}$ value for the $\mathrm{Chi}^{2}$ test of less than $0.1{ }^{27}$ If a very high heterogeneity was found (i.e., greater than $90 \%$ ) no meta-analyses were performed and results were described narratively.

We planned to investigate the publication bias using visual inspection for asymmetry of funnel plots if there were at least 10 studies included in the metaanalysis. We assessed the overall quality of the evidence for the primary outcome using the GRADE methodology. ${ }^{28,29}$ The GRADE approach uses five dimensions (risk of bias, consistency of effect, imprecision, indirectness and publication bias) to assess the quality of the body of evidence. The evidence is downgraded from high quality by one level if serious, or by two levels if very serious limitations are found for each of the five dimensions. We developed a summary of findings table presenting the quality of the evidence, reasons for limitation and main findings for the primary outcome in simple tabular format.

\section{Results}

\section{Study selection}

A total of 725 articles were retrieved from PubMed, 608 from EMBASE, and 185 from CENTRAL. After 175 duplicates were removed we were left 1343 articles and following a preliminary screening by reading the titles and abstracts we removed other 1331 articles. Out of 12 articles assessed as potentially eligible, 6 were excluded for the following reasons: two studies were not RCT, ${ }^{30,31}$ one study considered patients with recurrent urinary-tract infections and not acute pyelonephritis, ${ }^{32}$ two studies did not compare two regimens with the same antibiotic ${ }^{33,34}$ and one study used two different routes of administration; ${ }^{35}$ two further studies were considered awaiting assessment: one study was published in Chinese language $^{36}$ and one was a protocol in recruiting phase. ${ }^{37}$ We finally included 4 RCTs (Figure 1). 


\section{Characteristics of included studies}

We included 4RCTs ${ }^{23,38-40}$ with 439 participants conducted between 1987 and 2012. Three studies were conducted in European countries ${ }^{23,38,40}$ and one in the USA..$^{40}$ All studies included patients $\geq 15$ years with aPN. Three trials $\mathrm{s}^{38-40}$ included only patients with aPN, while the remaining trial ${ }^{23}$ included patients with complicated urinary tract infections; we extracted data only for the subgroups $(\mathrm{n}=34)$ with aPN. The majority of patients were women ( $87 \%$, range 66.7 to $100 \%$ ); mean age was 50.75 years, range 16-94.

One study ${ }^{23}$ enrolled only patients who were hos- pitalized, two studies ${ }^{38,39}$ included both inpatients and outpatients and the last one ${ }^{40}$ involved only outpatients. One study ${ }^{40}$ analyzed two subgroups of population treated with different antibiotic and, in this review, we classified it as Stamm A and Stamm B.

The antibiotic drugs investigated were the following: ampicillin, ${ }^{40}$ trimethoprim-sulfathoxazole, ${ }^{40} \beta$-lactams (pivampicillin/pivmecillinam); ${ }^{38}$ fluoroquinolones: fleroxacin ${ }^{23}$ and ciprofloxacinin. ${ }^{39}$

The duration of treatment for short antibiotic therapy ranged from 4 to 14 days. The duration for long therapy was $\geq 7$ days (ranging from 7 to 42 days), but at least 2 days longer than the corresponding short-

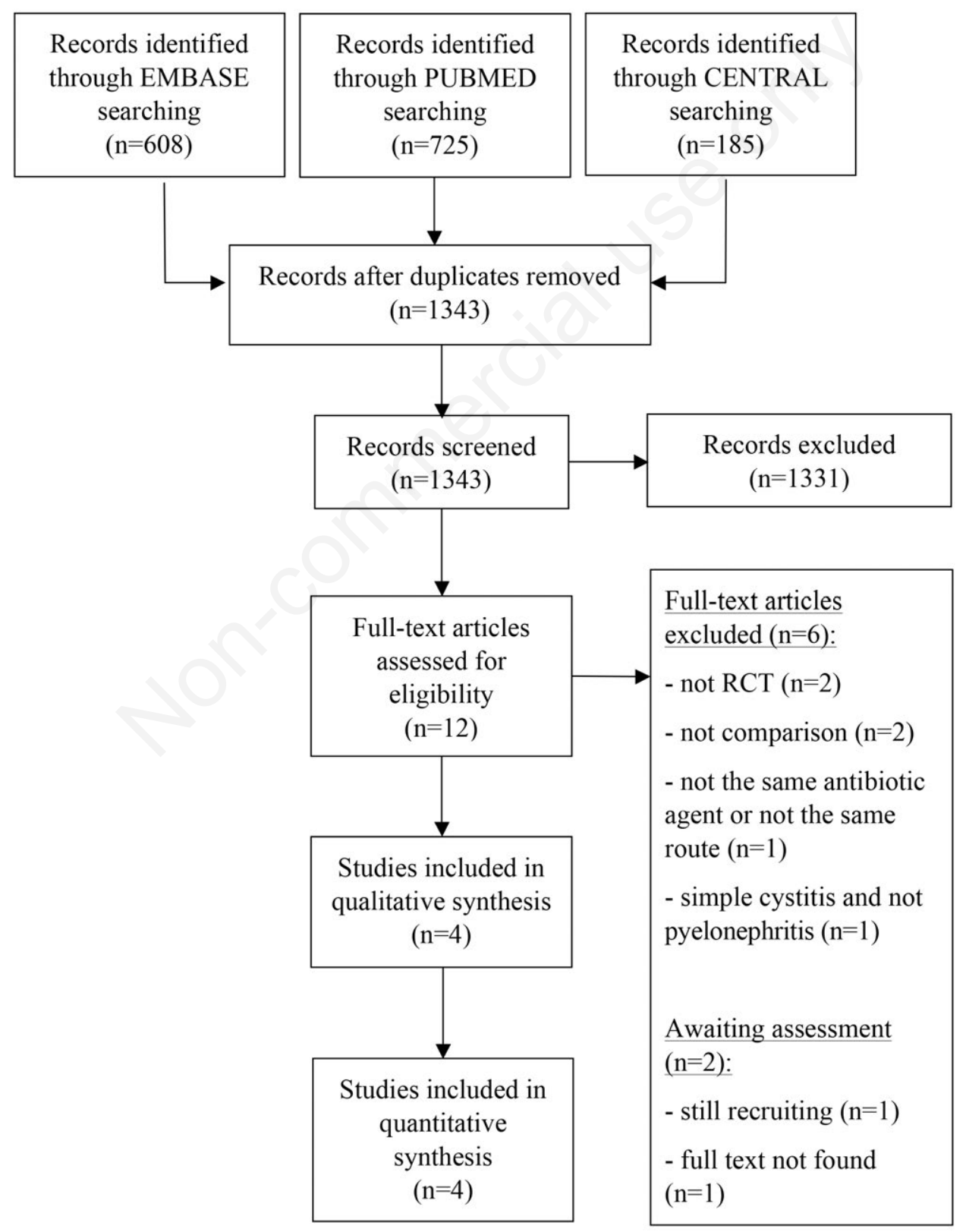

Figure 1. Study flow diagram. 
course treatment. The timing of outcomes assessment varied among studies and ranged from end of treatment up to 180 days.

Three trials were funded by pharmaceutics company: one from Hoffmann La Roche, ${ }^{23}$ one from Leo Pharmaceutical ${ }^{38}$ and one from Bayer. ${ }^{39}$ One trial was partially funded by National Institutes of Health and by a grant from Burroughs Wellcome Foundation ${ }^{40}$ (Table 1).

\section{Risk of bias assessment}

Three studies ${ }^{23,38,40}$ reported an appropriate method of randomization. One study ${ }^{39} \mathrm{did}$ not report sufficient information to permit judgment of low or high risk. Only one study ${ }^{39}$ reported an adequate procedure for allocation concealment, the others ${ }^{23,38,40}$ were at unclear risk of bias not reporting enough information to make a judgment. The main characteristics of the participants in the two groups were similar in all selected studies.

Two studies were double-blind (blinding of providers and patients) ${ }^{38,39}$ while the other two ${ }^{23,40}$ were open label and judged at high risk of performance bias. Two studies reported that the outcome assessors were blinded, ${ }^{23,39}$ one was open label and judged at high risk of detection bias ${ }^{40}$ while the fourth ${ }^{38}$ did not report sufficient information. All the studies were judged at high risk of attrition bias and at low risk of selective outcome reporting. Risk of bias assessment is presented in Figure 2. The risk of publication bias has not been evaluated because less than ten studies were included.

\section{Effects of interventions}

\section{Clinical success at the end of treatment}

We found no significant differences in clinical success between short and long antibiotic therapy at the end of treatment (RR 1.01, 95\% CI 0.96-1.07, 4 studies, 250 participants) with a moderate quality of evidence (Figure 3A and Table 2).

\section{Microbiological success at the end of treatment}

We found no significant differences in the microbiological success at the end of antibiotic treatment between short or long-term therapy (RR $0.99,95 \%$ CI 0.92-1.07, 2 studies, 190 participants) with low quality of evidence (Figure 3B and Table 2).

\section{Clinical relapse at 4-6 weeks}

We found no significant differences in clinical relapse at 4-6 weeks after the end of treatment between short and long-term therapy (RR 1.20, 95\% CI 0.43$3.30,2$ studies, 218 participants) with very low quality of evidence (Figure 4A and Table 2).

\section{Microbiological relapse at 4-6 week follow-up}

The long-term therapy seemed to prevent recurrences of the same biological germ up to 4-6 weeks after the end of treatment compared to short-term therapy (RR 2.39, 95\% CI 1.19-4.83, 2 studies, 95 participants) with very low quality of evidence (Figure 4B and Table 2).

\section{Microbiological reinfection at 4-6 week follow-up}

We found no significant differences between longand short-term therapy in the prevention of re-infection by different germs from the one originally isolated (RR 2.40, 95\% CI 0.68-8.49, 2 studies, 95 participants) with very low quality of evidence (Figure $4 \mathrm{C}$ and Table 2).

\section{Number of patients with adverse effects}

The number of patients with at least one adverse effect from antibiotic therapy seemed to be higher in the long-term therapy compared to that of short-term but the difference was not statistically significant (RR $0.63,95 \%$ CI $0.39-1.02,4$ studies, 375 participants)

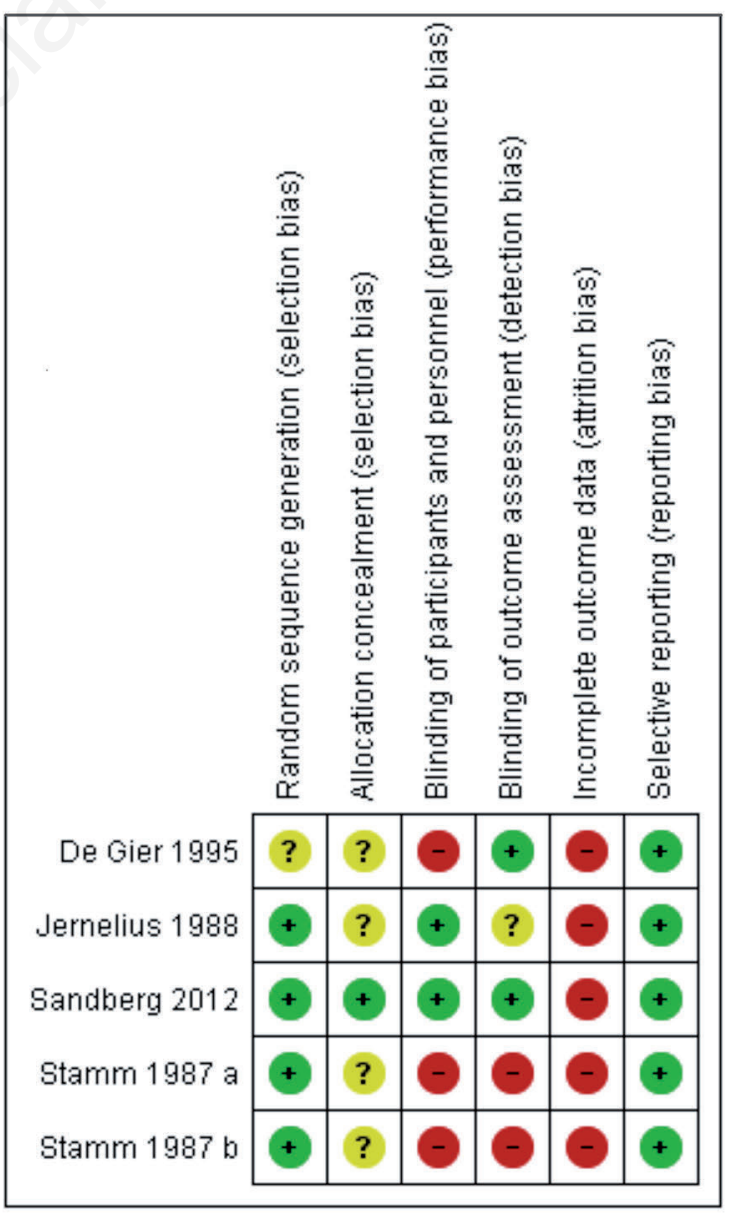

Figure 2. Risk of bias summary. 

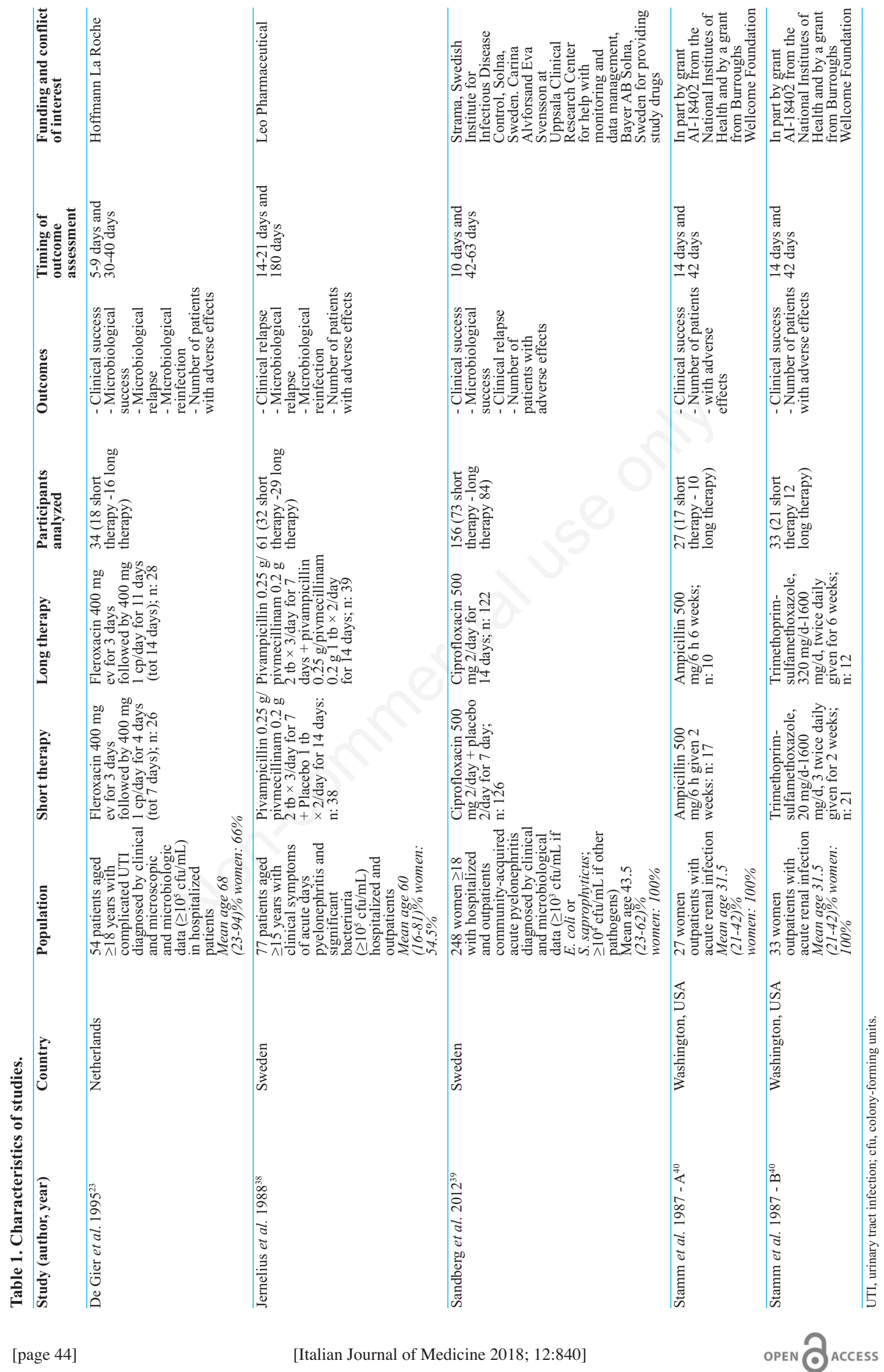
(Figure 5 and Table 2). None of the included studies reported results for the outcomes Renal impairment, Intensive Care Unit requirement, Mortality for any cause, Hospital readmission for the same cause.

\section{Discussion}

\section{Summary of main results}

This systematic review offers an update of results of all randomized trials that compare short- versus long-term antimicrobic therapy for acute pyelonephritis in adults. It is based on an extensive research, including incomplete data by attempting to contact all authors.

We found moderate quality evidence that shortand long-term treatments probably did not differ in terms of clinical success as well as in microbiological success at the end of treatment.

Conversely at 4-6 weeks after the end of treatment we found with a very low quality of evidence that microbiological relapse of the same biological germ may be more frequent with short-term therapy, but there may be no difference in the frequency of clinical relapse and re-infection by different germs.

Finally, we found that the incidence of any adverse effect seemed to be lower with the short-term therapy, though the results are not statistically significant and the quality of evidence is moderate. It was not possible to interpret the finding related to Mortality, Renal impairment, Intensive Care Unit requirement, Hospital readmission for the same cause since no data were reported in any of the included trials.

\section{Strengths, limitations and uncertainties}

The strengths of this review include the adherence to accepted standards for the conduct of systematic reviews and the use of extensive literature searches to identify relevant data. ${ }^{41-43}$ Moreover we included studies written in English, French, Spanish and Italian, so reducing the risk of language bias.

The major limitation of this review is the small number of participants on which we could base our conclusions: we found only four randomized studies fulfilling our inclusion criteria and most of them had had small sample size. Since aPN could be considered

A

\begin{tabular}{|c|c|c|c|c|c|c|c|c|}
\hline \multirow[b]{2}{*}{ Study or Subgroup } & \multicolumn{2}{|c|}{ short therapy } & \multicolumn{2}{|c|}{ long therapy } & \multirow[b]{2}{*}{ Weight } & \multirow{2}{*}{$\begin{array}{c}\text { Risk Ratio } \\
\mathrm{M}-\mathrm{H}, \text { Random, } 95 \% \mathrm{Cl}\end{array}$} & \multirow{2}{*}{$\begin{array}{c}\text { Risk Ratio } \\
\text { M-H, Random, } 95 \% \mathrm{Cl} \\
\end{array}$} & Risk of Bias \\
\hline & Events & Total & Events & Total & & & & A B C D E F \\
\hline De Gier 1995 & 11 & 18 & 11 & 16 & $1.2 \%$ & $0.89[0.54,1.46]$ & & $? ? \odot+\Theta$ \\
\hline Sandberg 2012 & 71 & 73 & 80 & 83 & $94.7 \%$ & $1.01[0.95,1.07]$ & & \\
\hline Stamm 1987 a & 11 & 17 & 4 & 10 & $0.4 \%$ & $1.62[0.70,3.73]$ & & \\
\hline Stamm 1987 b & 19 & 21 & 10 & 12 & $3.7 \%$ & $1.09[0.81,1.45]$ & & \\
\hline Total $(95 \% \mathrm{Cl})$ & & 129 & & 121 & $100.0 \%$ & $1.01[0.96,1.07]$ & 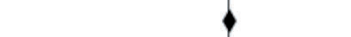 & \\
\hline Total events & 112 & & 105 & & & & & \\
\hline \multirow{2}{*}{\multicolumn{7}{|c|}{$\begin{array}{l}\text { Heterogeneity: } \operatorname{Tau}^{2}=0.00 ; \mathrm{Chi}^{2}=2.30, \mathrm{df}=3(\mathrm{P}=0.51) ; \mathrm{I}^{2}=0 \% \\
\text { Test for overall effect: } Z=0.43(\mathrm{P}=0.67)\end{array}$}} & 0.2 & \\
\hline & & & & & & & Favours long therapy Favours sh & \\
\hline
\end{tabular}

Risk of bias legend

(A) Random sequence generation (selection bias)

(B) Allocation concealment (selection bias)

(C) Blinding of participants and personnel (performance bias)

(D) Blinding of outcome assessment (detection bias)

(E) Incomplete outcome data (attrition bias)

(F) Selective reporting (reporting bias)

B

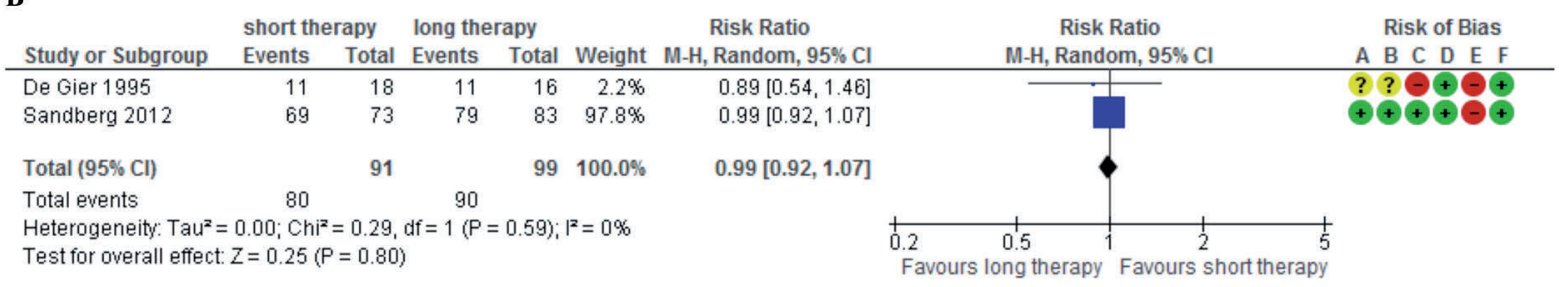

Risk of bias legend

(A) Random sequence generation (selection bias)

(B) Allocation concealment (selection bias)

(C) Blinding of participants and personnel (performance bias)

(D) Blinding of outcome assessment (detection bias)

(E) Incomplete outcome data (attrition bias)

(F) Selective reporting (reporting bias)

Figure 3. Forrest plots of comparison short vs long course antibiotic therapy at the end of treatment for A) clinical and B) microbiological success. 


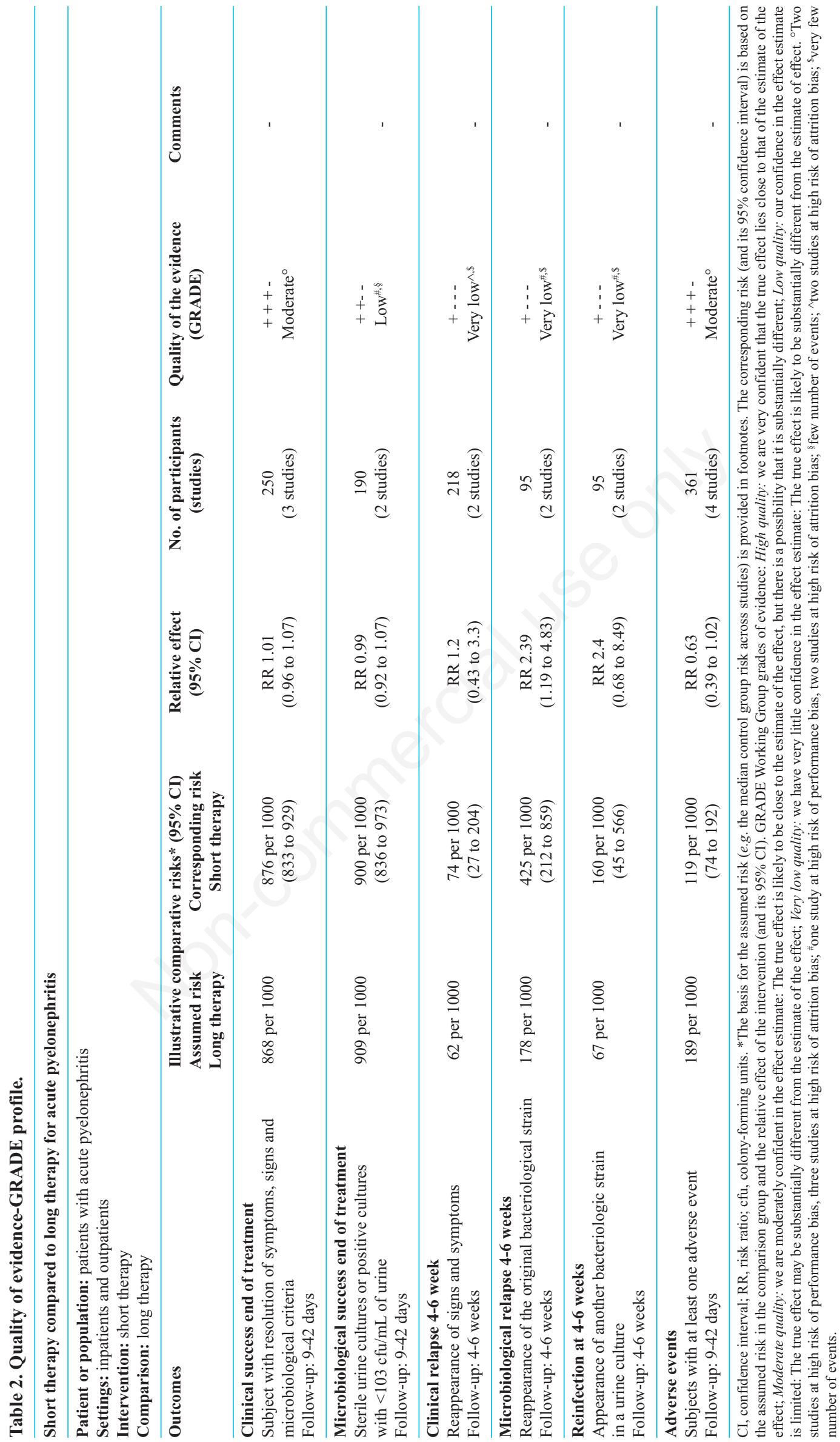


almost a rare condition because presented few cases every 10,000 habitants ${ }^{44}$ multicenter RCTs should be encouraged in order to increase the precision and the strength of the recommendation for the evidence of findings. Further studies should address this limitation by providing a larger sample size and improve the methodological quality of the studies, for example, opting for double-blind studies.

Furthermore, the retrieved evidence comes to studies - except Sandberg ${ }^{39}$ - that were $>10$ years old and some of the antibiotics used in the included studies (i.e., fleroxacin) are not available in a number of coun- tries worldwide. The included studies did not provide data on mortality and only on $\mathrm{e}^{40}$ reported information about the degree of severity of aPN.

In addition, we detected some deficiencies in the methodological quality of some of the included studies in this review. Only two trials were double blind and all were at high risk of attrition bias. Overall, the quality of evidence was judged from moderate to very low due to serious risk of bias and imprecision.

We could not assess the risk of publication bias by visual inspection for asymmetry of funnel plots because only four studies were included in meta-analy-

A

\begin{tabular}{|c|c|c|c|c|c|c|c|c|}
\hline \multirow[b]{2}{*}{ Study or Subgroup } & \multicolumn{2}{|c|}{ short therapy } & \multicolumn{2}{|c|}{ long therapy } & \multirow[b]{2}{*}{ Weight } & \multirow{2}{*}{$\begin{array}{c}\text { Risk Ratio } \\
\text { M-H, Random, } 95 \% \mathrm{Cl}\end{array}$} & \multirow{2}{*}{$\begin{array}{c}\text { Risk Ratio } \\
\text { M-H, Random, } 95 \% \mathrm{Cl}\end{array}$} & Risk of Bias \\
\hline & Events & Total & Events & Total & & & & A B C D E F \\
\hline Jernelius 1988 & 3 & 32 & 1 & 29 & $21.2 \%$ & $2.72[0.30,24.70]$ & & $\odot ?+? \odot+$ \\
\hline Sandberg 2012 & 5 & 73 & 6 & 84 & $78.8 \%$ & $0.96[0.31,3.01]$ & & 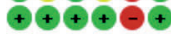 \\
\hline Total $(95 \% \mathrm{Cl})$ & & 105 & & 113 & $100.0 \%$ & $1.20[0.43,3.30]$ & & \\
\hline Total events & 8 & & 7 & & & & & \\
\hline \multicolumn{6}{|c|}{$\begin{array}{l}\text { Heterogeneity: } \operatorname{Tau}^{2}=0.00 ; \mathrm{Chi}^{2}=0.68, \mathrm{df}=1(\mathrm{P}=0.41) ; \mathrm{I}^{2}=0 \% \\
\text { Test for overall effect: } \mathrm{Z}=0.35(\mathrm{P}=0.73)\end{array}$} & & $\begin{array}{ccccc}0.05 & 0.2 & 1 & 5 & 20 \\
\text { Favours short therapy } & \text { Favours long therapy }\end{array}$ & \\
\hline \multicolumn{9}{|c|}{ Risk of bias legend } \\
\hline \multicolumn{9}{|c|}{ (A) Random sequence generation (selection bias) } \\
\hline \multicolumn{9}{|c|}{ (B) Allocation concealment (selection bias) } \\
\hline \multicolumn{9}{|c|}{ (C) Blinding of participants and personnel (performance bias) } \\
\hline \multicolumn{9}{|c|}{ (D) Blinding of outcome assessment (detection bias) } \\
\hline \multicolumn{9}{|c|}{ (E) Incomplete outcome data (attrition bias) } \\
\hline (F) Selective reportin & (reportin & bias) & & & & & & \\
\hline
\end{tabular}

B

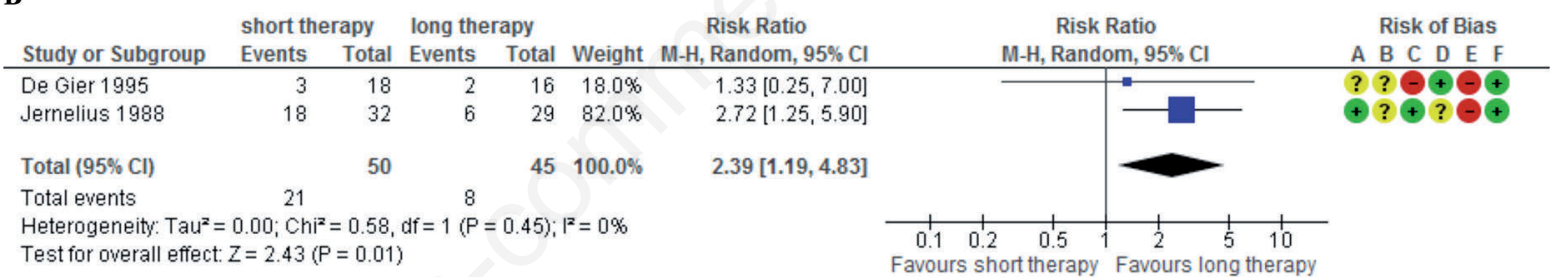

Risk of bias legend

(A) Random sequence generation (selection bias)

(B) Allocation concealment (selection bias)

(C) Blinding of participants and personnel (performance bias)

(D) Blinding of outcome assessment (detection bias)

(E) Incomplete outcome data (attrition bias)

(F) Selective reporting (reporting bias)

C

\begin{tabular}{|c|c|c|c|c|c|c|c|c|}
\hline \multirow[b]{2}{*}{ Study or Subgroup } & \multicolumn{2}{|c|}{ short therapy } & \multicolumn{2}{|c|}{ long therapy } & \multirow[b]{2}{*}{ Weight } & \multirow{2}{*}{$\begin{array}{c}\text { Risk Ratio } \\
\text { M-H, Random, } 95 \% \mathrm{Cl}\end{array}$} & \multirow{2}{*}{$\begin{array}{c}\text { Risk Ratio } \\
\text { M-H, Random, } 95 \% \mathrm{Cl}\end{array}$} & Risk of Bias \\
\hline & Events & Total & Events & Total & & & & $A B C D E F$ \\
\hline De Gier 1995 & 3 & 18 & 1 & 16 & $34.3 \%$ & $2.67[0.31,23.14]$ & & ? ? $\odot+\Theta \leftarrow$ \\
\hline Jernelius 1988 & 5 & 32 & 2 & 29 & $65.7 \%$ & $2.27[0.48,10.79]$ & & $\odot ?+? \odot \leftarrow$ \\
\hline Total $(95 \% \mathrm{Cl})$ & & 50 & & 45 & $100.0 \%$ & $2.40[0.68,8.49]$ & & \\
\hline Total events & 8 & & 3 & & & & & \\
\hline $\begin{array}{l}\text { Heterogeneity: Tau } \\
\text { Test for overall effect }\end{array}$ & $\begin{array}{l}0.00 ; \mathrm{Ch} \\
Z=1.35\end{array}$ & $\begin{array}{l}=0.01, \\
=0.18\end{array}$ & $d f=1(P$ & $0.90)$; & $\left.\right|^{2}=0 \%$ & & $\begin{array}{llll}0.05 & 0.2 & 1 & 5\end{array}$ & 20 \\
\hline
\end{tabular}

Risk of bias leqend

(A) Random sequence generation (selection bias)

(B) Allocation concealment (selection bias)

(C) Blinding of participants and personnel (performance bias)

(D) Blinding of outcome assessment (detection bias)

(E) Incomplete outcome data (attrition bias)

(F) Selective reporting (reporting bias)

Figure 4. Forest plots of comparison short vs long course antibiotic therapy at 4-6 weeks from the end of treatment for A) clinical relapse, B) microbiological relapse, and C) microbiological reinfection. 


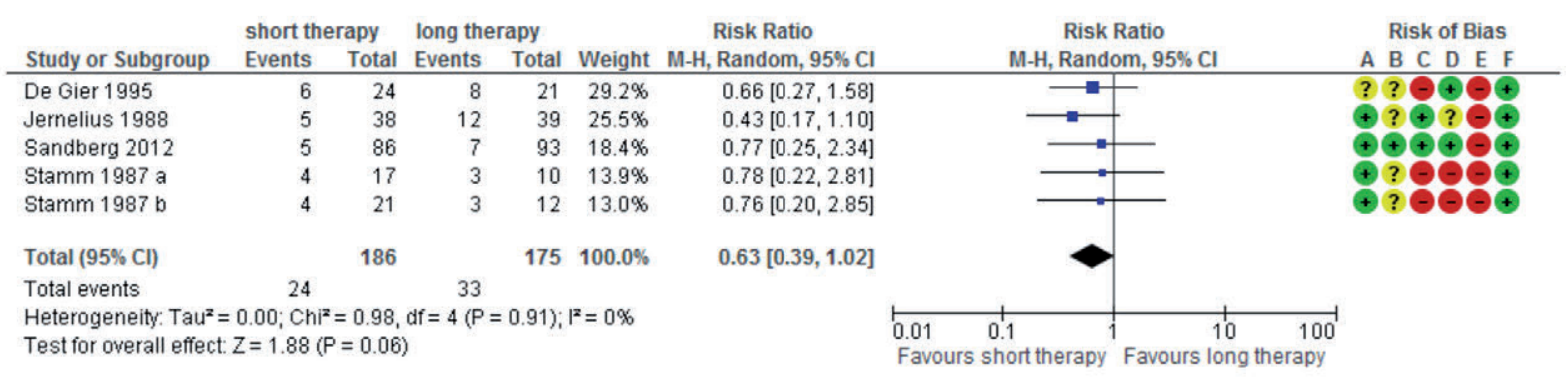

Risk of bias leqend

(A) Random sequence generation (selection bias)

(B) Allocation concealment (selection bias)

(C) Blinding of participants and personnel (performance bias)

(D) Blinding of outcome assessment (detection bias)

(E) Incomplete outcome data (attrition bias)

(F) Selective reporting (reporting bias)

Figure 5. Forest plot of comparison short vs long course antibiotic therapy, outcome: number of patients with adverse effects.

sis. So, the risk of publication bias cannot be excluded though we performed a very sensitive search looking also for unpublished studies and studies published in languages other than English.

\section{Agreements and disagreements with other studies or reviews}

We obtained the same findings of previous metaanalyses. ${ }^{24,45}$ The review published in 2008 by Kyriakidou et al. ${ }^{24}$ included 4 studies with 283 patients (one double-blinded RCT and three open-label RCTs) comparing a short (defined as 7-14 days) versus a long arm (defined as 14-42 days) for the same antibiotic treatment. Authors found no significant difference between the short- and long-course regimens efficacy at the end of treatment or at follow-up. Another systematic review published in 2013 by Eliakim-Raz et al. ${ }^{45}$ investigated the duration of treatment for acute pyelonephritis including 2515 patients, 1239 treated for $\leq 7$ days versus 1276 treated for $>7$ days. Five trials made comparisons using the same antibiotic, and three made comparisons using different antibiotics. They found out that seven days of treatment for acute pyelonephritis is equivalent to longer treatment in terms of clinical failure and microbiological failure, including bacteremic patients. In patients with urogenital abnormalities, the evidence, although weak, suggests that longer treatment is required.

Published Guidelines ${ }^{17}$ do not address the specific question of duration of treatment in acute pyelonephritis patients. However the following types of good clinical practice are performed: 7 days for fluoroquinolones, 10-14 days for $\beta$-lactams and 14 days for TMP-SMX. ${ }^{17-19}$ Since in our study we found no difference in terms of clinical and microbiological success or tolerability for short (from 4 to 14 days) and long course antibiotic therapy (ranging from 7 to 42 days), we can confirm the actual antibiotic recommendations even if the grade of recommendation is week for low quality of evidence due the paucity of patients and risk of bias of trials.

Finally, the reduction in patient exposure to antibiotics may also limit the increasing rates of antimicrobial drug resistance, ${ }^{46}$ decreasing costs, ${ }^{47}$ and may improve patient adherence and tolerability.

\section{Conclusions}

This review suggests that short-term treatment for acute pyelonephritis may be equivalent to longer-term treatment in terms of clinical success and microbiological success at the end of treatment adverse event may be slightly more frequent with the long therapy. The long-term therapy may be more effective for the prevention of microbiological relapse of the same biological germ up to 4-6 weeks after the end of treatment compared to short-term therapy.

Further high-quality research, through the launch of multicenter RCTs, is needed to confirm the clinical and microbiological equivalence of short and longerterm antibiotic treatment for aPN, including in patients with serious prognostic categories.

\section{References}

1. Foxman B, Klemstine KL, Brown PD. Acute pyelonephritis in US hospitals in 1997: hospitalization and In-hospital mortality. Ann Epidemiol 2003;13:144-50.

2. Stamm WE, Hooton TM. Management of urinary tract infections in adults. N Engl J Med 1993;329:1328-34.

3. Tibor F. Acute pyelonephritis. Available from: http:// emedicine.medscape.com/article/245559-overview Ac- 
cessed: August 11, 2015.

4. The Johns Hopkins Ambulatory Clerkship in Medicine. Dysuria. Available from: http://deptmed.med.som.jhmi. edu/ambclerk/disuria.htm Accessed: December 5, 2004.

5. Buonaiuto VA, Marquez I, De Toro I, et al. Clinical and epidemiological features and prognosis of complicated pyelonephritis: a prospective observational single hospital-based study. BMC Infect Dis 2014;14:639.

6. Foxman B, Gillespie B, Koopman J, et al. Risk factors for second urinary tract infection among college women. Am J Epidemiol 2000;151:1194.

7. Minardi D, D'Anzeo G, Cantoro D, et al. Urinary tract infections in women: etiology and treatment options. Int J Gen Med 2011;4:333-43.

8. Mnif MF, Kamoun M, Kacem FH, et al. Complicated urinary tract infections associated with diabetes mellitus: pathogenesis, diagnosis and management. Indian J Endocrinol Metab 2013;17:442-5.

9. Melekos MD, Naber KG. Complicated urinary tract infections. Int J Antimicrob Agents 2000;15:247-56.

10. Jolley JA, Kim S, Wing DA. Acute pyelonephritis and associated complications during pregnancy in 2006 in US hospitals. J Matern Fetal Neonatal Med 2012;25:2494-8.

11. Czaja CA, Scholes D, Hooton TM, Stamm WE. Population-based epidemiologic analysis of acute pyelonephritis. Clin Infect Dis 2007;45:273-80.

12. Strote SR, Klausner HA. High-dose, short-course levofloxacin review for complicated urinary tract infections and acute pyelonephritis. Available from: http://www.infectweb.com/only/artsrv2009_2.pdf

13. Singh N, Yu VL. Rational empiric antibiotic prescription in the ICU. Chest 2000;117:1496-9.

14. Foxman B, Ki M, Brown P. Antibiotic resistance and pyelonephritis. Clin Infect Dis 2007;45:281-3.

15. Brown P, Ki M, Foxman B. Acute pyelonephritis among adults: cost of illness and considerations for the economic evaluation of therapy. Pharmacoeconomics 2005; 23:1123-42.

16. Nicolle LE. AMMI Canada Guidelines Committee. Complicated urinary tract infection in adults. Can J Infect Dis Med Microbiol 2005;16:349-60.

17. Gupta K, Hooton TM, Naber KG, et al. International clinical practice guidelines for the treatment of acute uncomplicated cystitis and pyelonephritis in women: a 2010 update by the Infectious Diseases Society of America and the European Society for Microbiology and Infectious Diseases. Clin Infect Dis 2011;52:e103-20.

18. De Cueto M, Aliaga L, Alós J-I, et al. Executive summary of the diagnosis and treatment of urinary tract infection: guidelines of the Spanish society of clinical microbiology and infectious diseases (SEIMC). Enferm Infecc Microbiol Clin 2016 [Epub ahead of print].

19. Leggat P. Urinary tract infections - Therapeutic guidelines antibiotic, 13th ed. Melbourne: Australia Therapeutic Guidelines; 2006. pp 307-15.

20. Kang C, Kim K, Lee SH, et al. A risk stratification model of acute pyelonephritis to indicate hospital admission from the ED. Am J Emerg Med 2013;31:1067-72.

21. Huang ES, Stafford RS. National patterns in the treatment of urinary tract infections in women by ambulatory care physicians. Arch Internal Med 2002;162:41-7.

22. Kahan NR, Chinitz DP, Kahan E. Longer than recommended empiric antibiotic treatment of urinary tract in- fection in women: an avoidable waste of money. J Clinical Pharm Ther 2004;29:59-63.

23. De Gier R, Karperien A, Bouterb K, et al. A sequential study of intravenous and oral Fleroxacin for 7 or 14 days in the treatment of complicated urinary tract infections. Int J Antimicrob Agents 1995;6:27-30.

24. Kyriakidou KG, Rafailidis P, Matthaiou DK, et al. Shortversus long-course antibiotic therapy for acute pyelonephritis in adolescents and adults: a meta-analysis of randomized controlled trials. Clin Ther 2008;30:1859-68.

25. Higgins JPT, Altman DG, Sterne JAC, eds. Chapter 8: Assessing risk of bias in included studies. In: Higgins JPT, Green S, eds. Cochrane handbook for systematic reviews of interventions - Version 5.1.0 (updated March 2011). The Cochrane Collaboration, 2011. Available from: http://www.handbook.cochrane.org

26. DerSimonian R, Laird N. Meta-analysis in clinical trials. Control Clin Trials 1986; 7:177-88.

27. Higgins JP, Thompson SG, Deeks JJ, et al. Measuring inconsistency in meta-analyses. BMJ 2003;327:557-560

28. Oxman AD; GRADE Working Group. Grading quality of evidence and strength of recommendations. BMJ 2004;328:1490-4.

29. Guyatt G, Oxman AD, Akl EA, et al. GRADE guidelines 1. Introduction-GRADE evidence profiles and summary of findings tables. J Clin Epidemiol 2011;64:383-94.

30. Schaeffer EM. Re: Ciprofloxacin for 7 days versus 14 days in women with acute pyelonephritis: a randomised, open-label and double-blind, placebo-controlled, noninferiority trial. J Urol 2013;559-60.

31. Van der Starre WE, van Dissel JT, van Nieuwkoop C. Urinary tract infections. treatment duration of febrile urinary tract infections. Curr Infect Dis Rep 2011;13:571.

32. Crowley A. Therapy of recurrent invasive urinary-tract infections of men. N Engl J Med 1979;301:878-80.

33. Dawani ML, Sheikh MA, Naqvi SA. Clinical trial of norfloxacin in urinary tract infection. J Pak Med Assoc 1987;37:152-3.

34. Sanchez M, Collvinent B, Miró O, et al. Short-term effectiveness of ceftriaxone single dose in the initial treatment of acute uncomplicated pyelonephritis in women. A randomised controlled trial. Emerg Med J 2002;19:19-22.

35. Gelfand MS, Simmons BP, Craft RB, et al. A sequential study of intravenous and oral fleroxacin in the treatment of complicated urinary tract infection. Am J Med 1993;94:126S-30S.

36. Chen YJ, Wu JF. A single-blinded multicenter randomized controlled clinical trial of ceftizoxime sodium for injection in the treatment of lower respiratory tract and urine tract infections. Chinese J Infect Chemother 2008; 4:241-6.

37. Nieuwkoop C, van't Wout JW, Assendelft WJJ, et al. Treatment duration of febrile urinary tract infection (FUTIRST trial): a randomized placebo-controlled multicenter trial comparing short (7 days) antibiotic treatment with conventional treatment (14 days). BMC Infect Dis 2009;9:131.

38. Jernelius H, Zbornik J, Bauer CA. One or three weeks' treatment of acute pyelonephritis? A double-blind comparison, using a fixed combination of pivampicillin plus pivmecillinam. Acta Med Scand 1988;223:469-77.

39. Sandberg T, Skoog G, Hermansson AB, et al. Ciprofloxacin for 7 days versus 14 days in women with 
acute pyelonephritis: a randomised, open-label and double-blind, placebo-controlled, non-inferiority trial. Lancet 2012;380:484-90.

40. Stamm WE, McKevitt M, Counts GW. Acute renal infection in women: treatment with trimethoprim-sulfamethoxazole or ampicillin for two or six weeks. A randomized trial. Ann Intern Med 1987;106:341-5.

41. Chandler J, Churchill R, Higgins J, et al. Methodological standards for the conduct of new Cochrane Intervention Reviews. Methodological Expectations of Cochrane Intervention Reviews (MECIR); 2011. Available from: http://editorial unit.cochrane.org/sites/editorial

42. Liberati A, Altman DG, Tetzlaff J, et al. The PRISMA statement for reporting systematic reviews and metaanalyses of studies that evaluate healthcare interventions: explanation and elaboration. BMJ 2009;339.

43. Higgins JPT, Green S, eds. Cochrane Handbook for Systematic Reviews of Interventions - Version 5.1.0 [updated March 2011]. The Cochrane Collaboration, 2011.
Available from: http://handbook.cochrane.org

44. de Vrueh R, Baekelandt ERF, de Haan JMH. Background Paper 6.19 - Rare diseases. [Update on 2004 Background Paper]; 12 March 2013. Available from: http://www.who.int/medicines/areas/priority_medicines/BP6_19Rare.pdf

45. Eliakim-Raz N, Yahav D, Paul M, Leibovici L. Duration of antibiotic treatment for acute pyelonephritis and septic urinary tract infection-- 7 days or less versus longer treatment: systematic review and meta-analysis of randomized controlled trials. J Antimicrob Chemother 2013;68:2183-91.

46. Hu FP, Zhu DM, Fu W, et al. CHINET 2013 surveillance of bacterial resistance in China. Chin J Infect Chemother 2014;14:365-74.

47. Howard DH, Scott RD 2nd. The economic burden of drug resistance. Clin Infect Dis 2005;41:S283-6. 